\title{
Hypothesis to Explain Yawning, Cortisol Rise, Brain Cooling and Motor Cortex Involvement of Involuntary Arm Movement in Neurologically Impaired Patients
}

\author{
Simon BN Thompson ${ }^{1,2,3}$ \\ ${ }^{1}$ Faculty of Science and Technology, Bournemouth University, BH15 1YE, UK \\ ${ }^{2}$ Dementia Institute, Bournemouth University, BH1 3LT, UK \\ ${ }^{3}$ International Scientific Council for Research into Multiple Sclerosis, Universite Paris Ouest Nanterre La Défense, 92000 Nanterre, France
}

Corresponding author: Simon BN Thompson, Assistant Professor, Faculty of Science and Technology, Bournemouth University, Poole House (P305), Poole, BH12 5BB, UK, Tel: +44 1202 961558; E-mail: simont@bournemouth.ac.uk

Received: Nov 30, 2016; Accepted: Jan 06, 2017; Published: Jan 9, 2017

Citation: Thompson SBN. Hypothesis to Explain Yawning, Cortisol Rise, Brain Cooling and Motor Cortex Involvement of Involuntary Arm Movement in Neurologically Impaired Patients. J Neurol Neurosci. 2017, 8: 1.

\section{Abstract}

Association between the hormone cortisol and yawning has been found. The Thompson Cortisol Hypothesis proposed a link between yawning and rises in cortisol with further recent evidence from neuroscience showing communication between the motor cortex, brain-stem and hypothalamus. Hormonal and neuronal links form the proposed network that influences and also monitors cortisol and brain temperature regulation via the hypothalamus. Evidence supports the proposed connection between brain-stem, hypothalamus and motor cortex and further supports the Thompson Cortisol Hypothesis suggesting threshold levels of cortisol elicit yawning to cool the brain. Additionally, this may explain the well-known observed phenomenon known as parakinesia brachialis oscitans in brain-stem ischaemic stroke patients during involuntary yawning.

Keywords: Cortisol rise; Brain cooling; Motor cortex; Neurologically impaired patients

\section{Introduction}

Neurological disease is an umbrella term given to a range of diseases that encompass neurological pathways and their links with body structures and organs. It becomes particularly complicated when neurological pathways are shared and when they involve important brain structures that may have more than one responsibility within the body's neurological systems.

Neurological impairment occurs when the neuronal pathways are affected by disease either directly or indirectly and may be caused by a number of factors such as incomplete innervation resulting from a vascular stroke, disease to the nerves, infarction to surrounding tissues that include nerve endings and pathways, etc. Hormonal disruption can be an indirect cause of changing neuronal sensation or loss and it is important to consider the role of hormones within a particular pathway to understand subsequent impairment.

Cortisol is a naturally occurring hormone associated with immune protection and stress regulation [1,2]. Monitoring cortisol is helpful when stress levels are high, e.g. in adrenal insufficiency, Cushing's disease, and multiple sclerosis [1], where cortisol levels may fluctuate with fatigue.

Yawning and cortisol is associated with fatigue [3-5]. The Thompson Cortisol Hypothesis proposed the yawning reflex is triggered by threshold levels of cortisol to subsequently lower the temperature of the brain [6-8]. Yawning and cortisol, in combination, was proposed as a potential biomarker for early detection of neurological disease $[9,10]$ and the first electromyogram representation of the yawn was reported $[9,11]$ (Figures 1 and 2) [11].

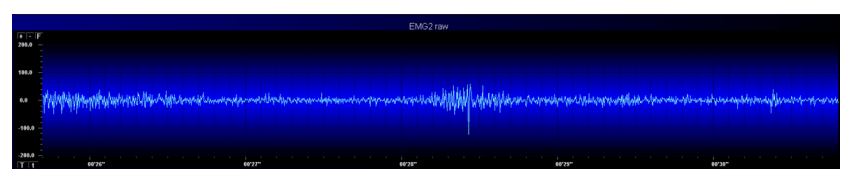

Figure 1 Electromyogram of the yawn reflex in humans.

Investigations using induced fatigue in healthy subjects have shown certain regions of the brain are activated during differing levels of cortisol production [12]. These provide support for the proposal that yawning is triggered by fluctuations in cortisol which affect the hypothalamus in order to facilitate brain cooling [7]. It has also been suggested that inhalation/exhalation may be an important factor in the yawning reflex and that perhaps this indirectly affects brain cooling with increased/decreased intake of oxygen.

The hypothalamus regulates temperature and circadian rhythm. Within the hypothalamus-pituitary-adrenal (HPA) axis, the hypothalamus produces enough cortisol releasing hormone to elicit release of adrenocortiocotropic hormone (from the pituitary gland) to protect against stress by activating the adrenal glands (zona fasciculate region) [13] to 
secrete cortisol and adrenaline [10]. It is thought that this in turn feeds back to the hypothalamus in order to lower brain temperature by the trigger of a yawn $[7,8,12,14]$.

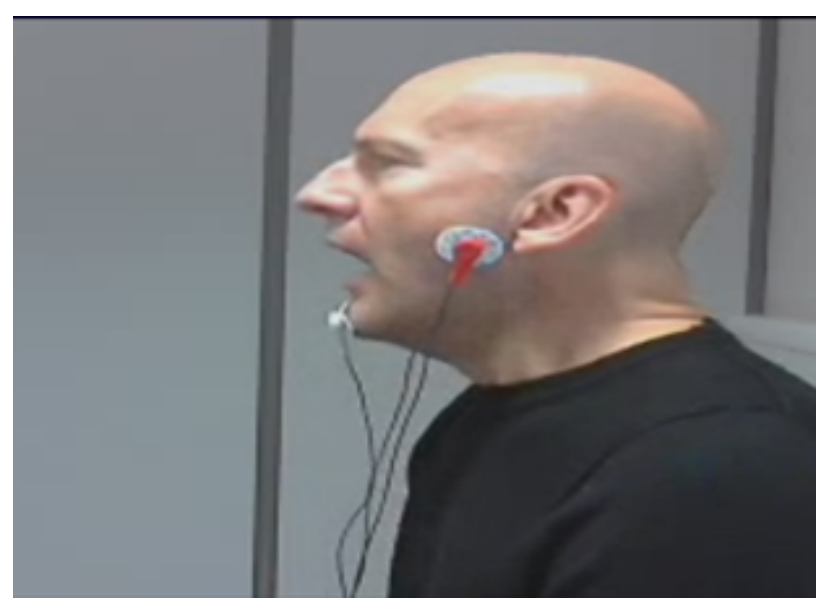

Figure 2 Electromyography monitoring and recording of the yawn reflex.

\section{The Hypothesis}

The Thompson Cortisol Hypothesis [6,15-17] is the first evidence-based report linking cortisol with yawning and shows that cortisol rises when yawning. The rise in cortisol is thought to trigger a yawn. Yawning may be the mechanism for controlling hormone regulation and for possibly regulating temperature in the hypothalamus.

The Thompson Cortisol Hypothesis was expanded [18] to explain brain temperature cooling and is now discussed in consideration of the motor cortex and how it may also explain the observed well-known phenomenon of involuntary movements in ischaemic brain-stem patients.

\section{Evaluation of The Hypothesis}

The motor cortex is most likely stimulated by cortisol production and needs to be communicating with the hypothalamus which regulates brain temperature. Since brain temperature may be lowered on yawning, it is suggested that the change in brain temperature is communicated via the hypothalamus to the motor cortex in order to stop yawning (Figure 1).

Alternative theories postulate cortisol produces tiredness which indirectly causes yawning. It is possible that yawning might resemble slow wave sleep and therefore, cortisol (associated with yawning), might increase tiredness especially in fatigue. The motor cortex may not be involved in eliciting yawning movement per se but acts as an inhibitor of limb movement as in the stroke-related phenomenon of parakinesia brachialis oscitans.

Gallup and Gallup have reported on symptom relief such as brain temperature reduction, in people with multiple sclerosis
$[19,20]$. Excessive yawning due to fatigue is a commonly observed symptom of multiple sclerosis [21].

As far back as 1923 the British Neurologist Sir Francis Walshe reported on brain-stem ischaemic stroke patients who raised the paralyzed arm when involuntarily yawning [22], subsequently known as parakinesia brachialis oscitans. This gave rise to considerable examination of strokes [23], and the proposition that the brain-stem may be acutely linked to the motor cortex $[23,24]$.

The brain-stem comprises the pons, mid-brain, and medulla oblongata [25], and is responsible for respiratory functioning and cardiac regulation. Researchers at the University of Adelaide [26] found plasticity of the human motor cortex in being influenced by time of day, and by circulating cortisol levels. The Gamma-aminobutyric acid (GABA)-beta receptors are often affected (Figure 3) [27] and particularly, they are influenced by the circulating cortisol levels [26].

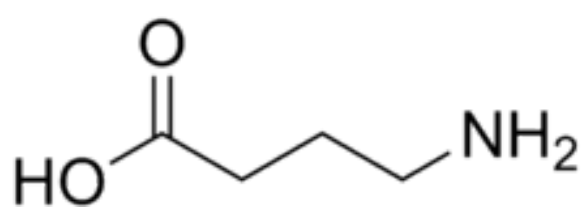

Figure 3 Schematic of the molecular structure of Gammaaminobutyric acid.

Hasan and colleagues [28] found the motor cortex in mice has sophisticated receptors important in learned motor responses. During motor learning, they found synaptic efficacy is enhanced between sensory and primary motor cortisol neurons. In humans, cortisol-specific receptor sites in the motor cortex may play a larger role in communicating between other regions such as the brain-stem and hypothalamus.

Communication between these sites may be linked less intimately by neural networks but by hormone systems (e.g. HPA-axis). This would provide a possible understanding of why brain-stem lesion stroke patients are able to raise the affected arm when yawning because of threshold cortisol levels triggering yawning and detected by motor cortex receptors. These receptors act on the motor end plates to cause muscle movement in the arm.

It is hypothesised that cortisol plays an important role in indirectly regulating the movement of limbs via feedback to the motor cortex and more precisely, via the hypothalamus communicating with the motor cortex (Figure 4) [7].

It is suggested that the brain-stem fails to act on changes in cortisol levels permitting upper limb musculature, signalled by the motor cortex due to rises in cortisol, to contract and raise the upper limb. In stroke patients, cortisol levels may be inadequately detected together with incomplete innervation and disrupted proprioception, resulting in movement of the paralyzed limb [29]. In contrast, on the unaffected side of the body, movement is inhibited because the rise in cortisol "signal" is recognised within the brain-stem. 


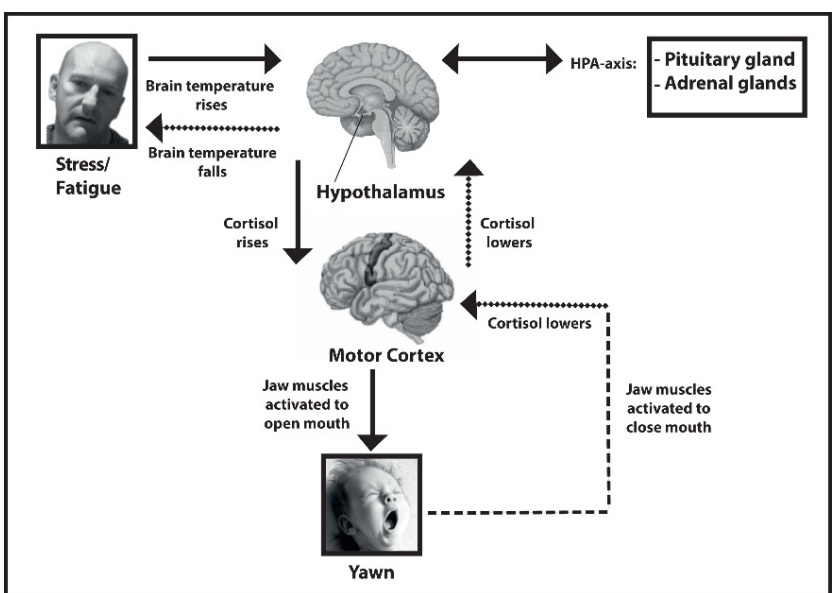

Figure 4 Jaw muscle activation and yawning due to cortisol rises.

Comparison between pathways (Figures $\mathbf{4}$ and 5) allows postulation that the brain-stem is involved in inhibiting movement. Reacting to cortisol levels detected by the hypothalamus, the brain-stem inhibits upper limb movement when it is not required to move; failure to inhibit may be parakinesia brachialis oscitans [30] following brain-stem ischaemic stroke.

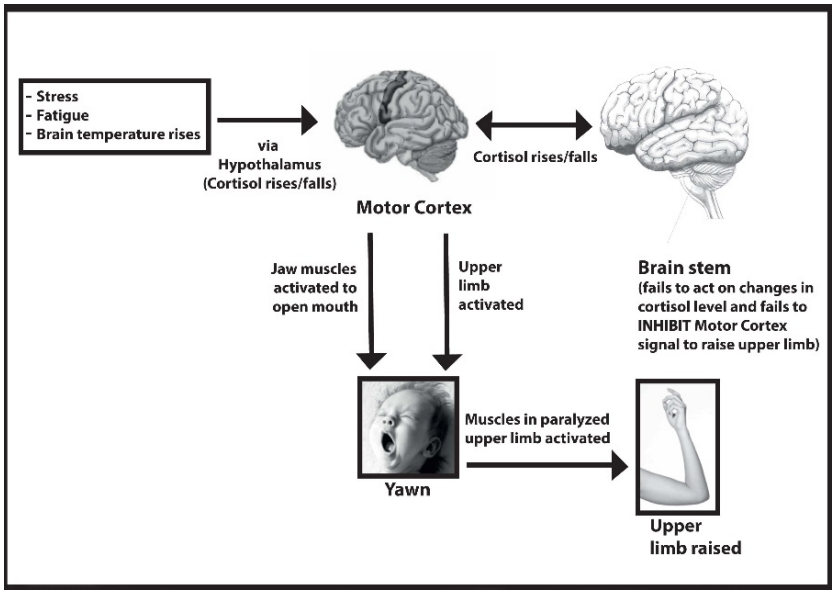

Figure 5 pathway showing Paralyzed upper limb rise in ischaemic stroke.

It is accepted that hormones do not act singularly; however, it is plausible that in brain-stem ischaemia it is the loss of neuronal detection systems that would normally communicate with the motor cortex that are responsible for the unusual movement seen in stroke patients. It is plausible that the detection system relies on monitoring cortisol levels, which when faulty, result in yawning without recognizing falling levels of cortisol after yawning.

\section{Empirical Data}

Fatigue has been found with elevated salivary cortisol levels in elite tennis players [30]. 'Fatigue' has often been defined in terms of tiredness due to physical exercise but can also be observed in mental concentration on tasks demanding prolonged alertness. Some researchers believe that brains are more efficient when cooler and therefore, a deep breath of fresh air can cool an overheated brain [31].

From fMRI studies, yawning seems to involve frontal and parietal lobes, insula and amygdala [32]. It is probable there is a threshold level of cortisol to be reached following fatigue, empathy, or sleep deprivation, to elicit yawning. Electromyography (EMG) nerve activity in the jaw muscles increases with yawning and is associated with cortisol level rises $[11,18]$. EMG feedback and changes in hormone levels within the HPA-axis continually regulate cortisol and adrenaline production within the closed feedback loop. The motor cortex is likely to be implicated since it controls movement including activating the muscles along the jaw-line.

In multiple sclerosis, incomplete innervation due to loss of myelin sheath around nerves is largely responsible for uncoordinated movements. Temperature rises are coexistent with fatigue and excessive yawning $[33,34]$. It is postulated that levels of cortisol rise when people yawn with multiple sclerosis just as with healthy people [1,35], but at higher levels than healthy people because of excessive fatigue [33].

\section{Consequences of The Hypothesis and Discussion}

Evidence suggests that cortisol may trigger the yawning reflex when sufficient threshold is reached. The association between fatigue and yawning, fatigue and cortisol, and between yawning and cortisol, firmly suggests that extra load in terms of physical and/or mental exertion creates a stress response in the form of cortisol release within the HPA-axis.

The proposal that cortisol levels rise to trigger a yawn is supported by evidence that suggests the hormone is responsible also in the reduction of brain temperature especially where rises in temperature are frequently observed with fatigue as in multiple sclerosis.

The suggestion that cortisol is also implicated in the involuntary movement observed in ischaemic stroke patients arises from the fact that motor cortex receptors are switched on for cortisol detection and monitoring and that because yawning and cortisol is associated, it is probable that yawning indirectly signals the paralyzed arm to rise because of irregulation in cortisol levels and damage to neuronal inhibitory pathways that affect gross motor movement, particularly in the upper arm.

\section{Conclusion}

The author is conducting research that focuses on cortisol changes in people with multiple sclerosis to see if yawning 
determines brain temperature reduction as a consequence of cortisol levels rising during fatigue. It is anticipated that cortisol levels may be higher than those seen in healthy participants and that brain temperature fluctuations may depend on fatigue and the stage of the disease.

Yawning is a primitive response seen in many vertebrates and has been reported as far back as $400 \mathrm{BC}$ by Hippocrates in his book De Flatibus Liber [A Treatise on Wind] [36]. His observation that yawning may expel hot air from our mouths to lower temperature, although simply described within the science of the time, may have some foundation on our current thinking about the effects of brain structures.

In particular, on our understanding of the functions of the hypothalamus on prevention of brain overheating, performance of athletes, and the everyday welfare of all of us in society with and without neurological disease. Yawning is indeed a primitive yet profound behaviour that has been overlooked by scientists for centuries.

\section{Sources of Support}

The empirical work is supported by Research Incentive Grants amounting to $£ 4,000$ provided by Bournemouth University, UK.

\section{Conflicts of Interest}

None.

\section{References}

1. Thompson SBN, Bishop P (2012) Born to yawn? Understanding yawning as a warning of the rise in cortisol levels: Randomized trial. Interact J Med e4: 1-9.

2. Thompson SBN (2014) Yawning, fatigue, and cortisol: Expanding the Thompson Cortisol Hypothesis. Med Hyp 4: 494-496.

3. Walker S, Taipale RS, Nyman K, Kraemer WJ, Hakkinen K (2011) Neuromuscular and hormonal responses to constant and variable resistance loadings. Med Sci Sports Exer 1: 26-33.

4. Bresciani G, Cuevas MJ, Molinero O, Almar M, Suay F, et al. (2011) Signs of overload after an intensified training. Int J Sports Med 2011;32;5: 338-343.

5. Locke, S, Osborne, M, O'Rourke P (2011) Persistent fatigue in young athletes: measuring the clinical recovery and identifying variables affecting clinical recovery. Scandin J Med Sci Sports 1: 90-97.

6. Thompson SBN (2010) The dawn of the yawn: Is yawning a warning? Linking neurological disorders. Med Hyp 75: 630-633.

7. Thompson SBN (2015) Pathways to yawning: Making sense of the Thompson Cortisol Hypothesis. Med Res Arch 3: 1-7.

8. Thompson SBN, Richer S (2015) How yawning and cortisol regulates the attentional network. J Neurosci Rehab 1: 1-9.

9. Thompson SBN (2015) Yawning and cortisol as a potential biomarker for early detection of multiple sclerosis. Int J Med Health Biomed Bioeng Pharma Eng 5: 382-386.
10. Thompson SBN (2016) Diagnostic biomarkers - Exploring the potential of cortisol and yawning in the detection of neurological disease processes. J Neurol Neurosci 7: 112.

11. Thompson SBN (2013) How to catch a yawn: Initial observations of a randomized controlled trial. WMC Neurol 8: 1-8.

12. Thompson SBN, Daly S, Le Blanche A, Adibi M, Belkhiria C, et al. (2016) fMRI randomized study of mental and motor task performance and cortisol levels to potentiate cortisol as a new diagnostic biomarker. J Neurol Neurosci 7: 92.

13. Schillings WJ (2008) Physiology and tests of adrenal cortisol function. Glob Lib Wom Med.

14. Thompson SBN (2015) Health psychology intervention Identifying early symptoms in neurological disorders. Int J Med Health Biomed Bioeng Pharma Eng 4: 351-355.

15. Thompson SBN: Born to yawn? Cortisol linked to yawning: a new hypothesis Med Hyp 77: 861-862.

16. Thompson SBN, Frankham C, Bishop P (2014) The art of capturing yawn using the science of nerve impulses and cortisol levels in a randomized controlled trial. Thompson Cortisol Hypothesis as a potential predictor of neurological impairment. Int J Arts Sci 3: 529-543.

17. Thompson SBN, Rose K, Richer S (2014) Yawning with cortisol: Examining the neuroscience behind the Thompson Cortisol Hypothesis for supporting rehabilitation of neurologically impaired individuals. J Neurosci Rehab 1: 1-11.

18. Thompson SBN (2014) Yawning, fatigue and cortisol: Expanding the Thompson Cortisol Hypothesis. Med Hyp 4: 494-496.

19. Gallup AC, Gallup GG (2007) Yawning as a brain cooling mechanism: Nasal breathing and forehead cooling diminish the incidence of contagious yawning. Evol Psychol 1: 92-101.

20. Gallup AC, Gallup GG (2010) Yawning, sleep, and symptom relief in patients with multiple sclerosis. Sleep Med 11: 329-330.

21. Fleming WE, Pollak CP (2005) Sleep disorders in multiple sclerosis. Sem Neurol 25: 64-68.

22. Provine $R$ (2012) Curious behaviour: Yawning, laughing, hiccupping and beyond. New York, USA: Belknap pp: 31-32.

23. Walusinski O, Neau JP, Bogousslavsky J (2010) Hand up! Yawn and raise your arm. Int J Stroke 5: 21-27.

24. Thompson SBN (2011) Is yawning a warning, neurologically? WMC Neurol 2: 1-7.

25. Thompson SBN (2012) Neuroanatomy - A beginner's guide. Saarbruken: LA.

26. Sale MV, Ridding MC, Nordstrom MA (2008) Cortisol inhibits neuroplasticity in human motor cortex. J Neurosci 33: 8285-8293.

\section{Wikipedia (2016) GABA receptor: Accessed 19.07.2016.}

28. Hasan MT, Hernández GS, Dogbevia G, Treviño M, Bertocchi I, et al. (2013) Role of motor cortex NMDA receptors in learningdependent synaptic plasticity of behaving mice. Nat Comm 2258: 1-9.

29. Wimalaratna HSK, Capildeo R (1988) Is yawning a brain stem phenomenon? Lancet 8580: 300.

30. Gomes RV, Coutts AJ, Viveiros L, Aoki MS (2011) Physiological demands of match-play in elite tennis: A case study. Europ J Sport Sci 2: 105-109. 
31. Gallup JrGG, Gallup AC (2009) Excessive yawning and themoregulation: Two case histories of chronic, debilitating bouts of yawning. Sleep Breath.

32. Krestel H, Weisstanner C, Hess C, Bassetti C, Nirkko A, et al. (2013) Insular and caudate lesions release abnormal yawning in stroke patients Brain Struct Funct.

33. Gallup AC, Gallup GG (2008) Yawning and thermoregulation. Physiol Beh 95: 10-16.
34. Gallup A, Eldakar O (2013) The thermoregulatory theory of yawning: What we know from over 5 years of research Front Neurosci 6: 1-13.

35. Thompson SBN, Simonsen M (2015) Yawning as a new potential diagnostic marker for neurological diseases. J Neurol Neurosci 6: 22.

36. Vigier MJ (1620) (translated by) Les aphorismes d'Hippocrate. JA Huguetan, Lyon, France. 